\title{
New Precision Measurement of Muonium Hyperfine Structure
}

\author{
Y. Ueno*, Y. Matsuda, S. Seo, T. Tanaka, H. A. Torii, D. Yagi, H. Yasuda \\ University of Tokyo \\ E-mail: yueno@radphys4.c.u-tokyo.ac.jp
}

\section{S. Nishimura, K. Sasaki, Y. Sato, K. Shimomura, P. Strasser, H. Yamaguchi} $K E K$

S. Kanda

RIKEN

K. S. Tanaka

Tohoku University

T. Yamanaka

Kyushu University

\section{for MUSEUM collaboration}

\begin{abstract}
Muonium is one of the best probes for the test of the bound-state QED, owing to the absence of the effect from the internal structure of nucleons. The recent establishment of intense pulsed muon beams at Japan Proton Accelerator Research Complex (J-PARC) paves the way to improved measurement of the muonium ground-state hyperfine structure (MuHFS). The measurement of MuHFS also determines the muon magnetic moment precisely, which is an important input parameter for the measurements of muon $\mathrm{g}$-2, which is known for the $3 \sigma$ discrepancy between the experiment and the theory. Muonium Spectroscopy Experiment Using Microwave (MuSEUM) collaboration aims to improve the precursor MuHFS experiment by a factor of ten. We present the status of the project and the prospect.
\end{abstract}

The 39th International Conference on High Energy Physics (ICHEP2018)

4-11 July, 2018

Seoul, Korea

${ }^{*}$ Speaker. 


\section{Motivation and Method}

Muonium is the bound state of a positive muon and an electron. Unlike ordinary atoms such as hydrogen, muonium is free from the internal-structure effect of nucleons, and theorists can calculate its energy levels very precisely. By comparing the experimental result of the muonium ground-state hyperfine structure (MuHFS) spectroscopy and the theoretical calculation, one can test the boundstate QED with high precision. Muonium Spectroscopy Experiment Using Microwave (MuSEUM) collaboration aims a new measurement of the MuHFS using intense pulsed muon beams at Japan Proton Research Accelerator Complex (J-PARC).

There are two different methods for the measurements of MuHFS; one in a low magnetic field and one in a high field. The best measurement results of the low-field method and the high-field method are [1,2],

$$
\begin{aligned}
\Delta v_{\mathrm{LF}} & =4.4633022(14) \mathrm{GHz}(300 \mathrm{ppb}), \\
\Delta v_{\mathrm{HF}} & =4.463302776(51) \mathrm{GHz}(12 \mathrm{ppb}),
\end{aligned}
$$

where ppb means parts per billion, $10^{-9}$. The uncertainties of both measurements are dominated by the statistical uncertainty, thus using an intense pulsed beam at J-PARC is important for further improvement. Currently, the measurement in a low magnetic field is ongoing in the existing beamline D2 at J-PARC, and once J-PARC completes the construction of a new beamline H1, we will start a measurement in a high field there. We expect to improve the MuHFS by a factor of ten, with 100 days of physics run using the beamline H1.

The theoretical value including the contribution of hadronic and weak interactions is [3]

$$
\Delta v_{\text {th }}=4.463302868(271) \mathrm{GHz}(61 \mathrm{ppb}),
$$

where the main source of the uncertainty is the uncertainty of the muon mass $m_{\mu}$, which is determined by experiments. A new spectroscopy of muonium 1S-2S can reduce this uncertainty [4]. Combined with such measurements, MuSEUM can contribute to the test of the bound-state QED.

Another motivation of the MuHFS measurement is the synergy with new experiments of the muon anomalous magnetic moment. The muon anomalous magnetic moment $\left(a_{\mu}=\frac{(g-2)}{2}\right.$, where $g$ is the muon $g$-factor) is known for more than $3 \sigma$ discrepancy between the measurement using a muon storage ring at the Brookhaven National Laboratory (BNL) [5] and the theoretical calculation of the Standard Model (SM) [6]. New storage ring experiments are proposed at Fermilab [7] and J-PARC [8]. They aim measurements of $a_{\mu}$ with a precision of $100 \mathrm{ppb}$, which is approximately a factor of five improvement. To extract the $a_{\mu}$ from a storage-ring experiment, one needs the muon magnetic moment $\mu_{\mu}$ as an external parameter.

The most precise value of $\mu_{\mu}$ is determined from the MuHFS measurement in high field [2] with a precision of $120 \mathrm{ppb}$. There is an alternative way of determination of the $\mu_{\mu}$, with an assumption that the theoretical calculation of MuHFS is correct. The latter way yields $\mu_{\mu}$ with a precision of 27 ppb. MuSEUM collaboration can improve the MuHFS measurement, and determines $\mu_{\mu}$ with a precision of $12 \mathrm{ppb}$, without assuming the MuHFS calculation is correct; thus MuSEUM can fully exploit the results of the new storage ring experiments.

Here we briefly introduce the measurement procedure. The procedure is described in detail elsewhere [9]. Figure 1 shows the schematic view of the experimental apparatus. We inject 100\% 
spin-polarized muons into highly pure ( $>99.995 \%)$ krypton gas, and then muoniums are formed by electron capture. Microwave in cavity induces the spin flip of the muons, which is detected by the positron asymmetry measurement.

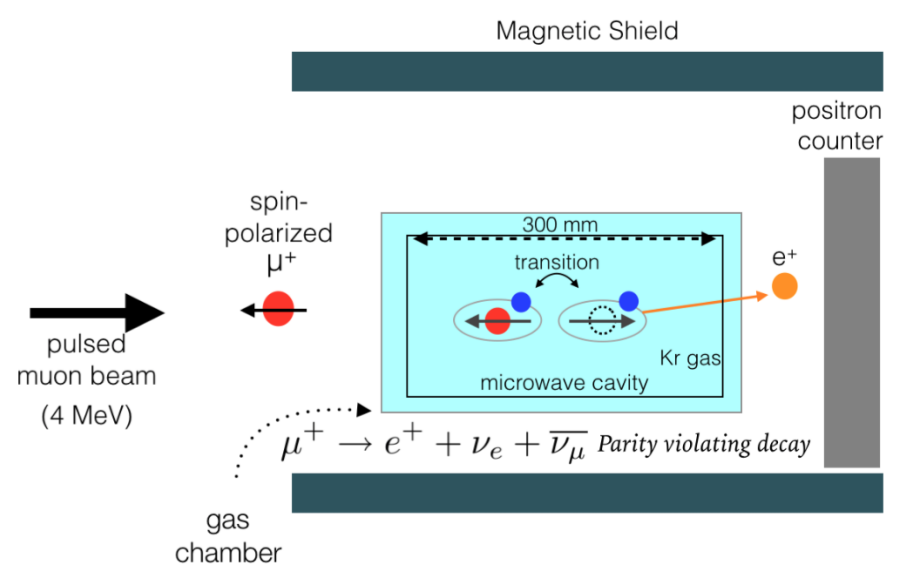

Figure 1: Schematic diagram of the experimental procedure.

\section{Spectroscopy in Krypton with Lower Pressures}

The dominant source of the uncertainty in the precursor measurement is statistics. One of the most dominant systematic uncertainties is the uncertainty related to the collision between muonium and krypton atoms. The collision shifts the MuHFS frequency, so one needs to measure the MuHFS at two or more different krypton pressures and extrapolate the results to the value at zero density. Measurements at lower krypton gas pressures can reduce the uncertainty related to the extrapolation. Such measurements, however, lose the statistics due to the broadening of the muonium distribution in the krypton gas resulting the large portion of the muons stops in the cavity wall.

Since we have observed the first MuHFS resonance in June 2016, we have conducted several measurements in low magnetic field. All of them are at krypton pressure at 1.0 atmosphere (atm). Recently, we developed a new microwave cavity with a larger volume, which enables the measurement at lower krypton gas pressure without severe loss of the statistics mentioned above.

In the measurement on March and June 2018, we have observed the resonances at krypton pressures at $0.3,0.4$, and $0.7 \mathrm{~atm}$. Figure 2 shows one of the obtained resonance lines at $0.4 \mathrm{~atm}$. The signal is defined by a difference of the numbers of positrons counted when the microwave is on and off. The precursor measurements $[1,2]$ used krypton pressure higher than 0.7 atm, so it is a promising result for reducing the systematic uncertainty from the collisional shift of the MuHFS. We expect to reduce such uncertainty to $9 \mathrm{~Hz}$ (2 ppb), while the precursor experiment [2] has estimated the uncertainty as $22 \mathrm{~Hz}(5 \mathrm{ppb})$.

The analysis in detail is ongoing, but we expect the lack of statistics as the primary source of the uncertainty of the measurement in 2018. J-PARC plans to enhance the proton beam power by a factor of two, and there is the new beamline $\mathrm{H} 1$ under construction, which will enable ten times more intense muon beam. 


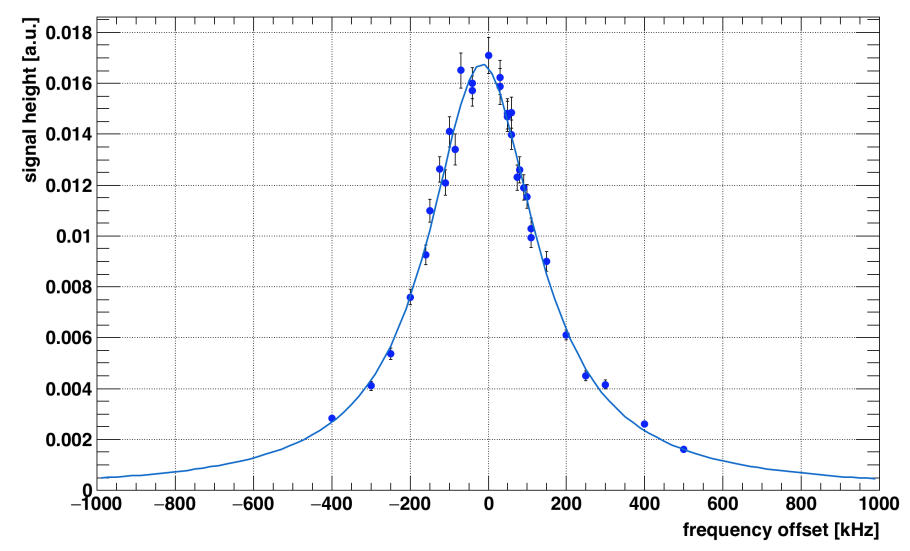

Figure 2: A typical resonance observed when the krypton gas pressure is 0.4 atomosphere. The horizontal axis is the relative frequency in $\mathrm{kHz}$. The vertical axis is the signal height. Blue line indicates the fitted Lorentz function.

\section{Conclusion and Future Prospect}

We have reported the recent status of the MuSEUM. We succeeded in the measurement with lower krypton gas pressures, which enables us to suppress the systematic uncertainties related to the collision between a muonium and krypton atoms. For the measurement on June 2018, the proton beam power at J-PARC is $500 \mathrm{~kW}$, and the beam power will be enhanced to $1 \mathrm{MW}$ shortly. The new beamline $\mathrm{H} 1$ will ameliorate the available number of the muons by another factor of ten.

\section{References}

[1] D. E. Casperson, et al., A New High Precision Measurement of the Muonium Hyperfine Structure Interval $\Delta v$, Phys. Lett., B59, 4 (1975)

[2] W. Liu, et al., High Precision Measurements of the Ground State Hyperfine Structure Interval of Muonium and of the Muon Magnetic Moment, Phys. Rev. Lett., 82, 711(1999)

[3] P. J. Mohr, D. B. Newell, and B. N. Taylor, CODATA recommended values of the fundamental physical constants: 2014, Rev. of Mod. Phys., 88, 035009 (2016)

[4] P. Crivelli, The Mu-MASS experiment, arXiv [physics.atom-ph/1811.00310] (2018)

[5] G. W. Bennett, et al., Final report of the E821 muon anomalous magnetic moment measurement at BNL, Phys. Rev., D73, 072003 (2006)

[6] K.A. Olive et al., (Particle Data Group), Review of Particle Physics, Chin. Phys., C38, 090001 (2014)

[7] J Holzbauer, The Muon g-2 Experiment Overview and Status as of June 2016, Jour. of Phys.: Conf. Ser. 770012038 (2016)

[8] M. Otani, Status of the Muon g-2/EDM Experiment at J-PARC (E34), JPS Proc. Conf., 8, 025008 (2015)

[9] P. Strasser, et al., New Precision Measurement of Muonium Hyperfine Structure, Hyp. Int. 237, 124 (2016) 